\title{
Torneo criminal, aversión al riesgo y seguridad ciudadana: comportamiento criminal en Bogotá D.C.*
}

\section{Criminal Tournament, Risk Aversion and Public Safety: Criminal Behavior in Bogotá D.C.}

\author{
Manfred Grautoff*, Fernando Chavarro ${ }^{* * * * *}$, Jazmín Balaguer ${ }^{* * * * * *}$
}

\section{Resumen}

Este documento analiza las políticas públicas de seguridad en la ciudad de Bogotá, formuladas sobre el diagnóstico que correlaciona la criminalidad con las causas objetivas del conflicto. La evidencia empírica de la revisión bibliográfica demuestra que dicha relación no es significativa; por esto se desarrolló un modelo de comportamiento criminal que explique el fenómeno como una competencia donde el delincuente compite con otros individuos, lo que forma una estructura que mejora la productividad de la cadena criminal, eliminando a los agentes menos aptos en actividades criminales. La evidencia empírica es preliminar debido a que no se tiene acceso a los micro-datos de la encuesta de victimización de 2003, los cuales han sido declarados reserva estadística por parte del Gobierno de Colombia.

Palabras clave: Criminalidad, Windows Broken, Teoría racional del crimen, Teoría del torneo, aversión al riesgo.

Glasificación JEL: A12, B21, C61, C73.

* Documento de avance de investigación, perteneciente a la línea de desarrollo económico y economía de defensa, que vienen adelantando los autores.

* Economista, magíster en Economía, magíster en Seguridad y Defensa Nacionales. Investigador, docente Universidad de La Sabana. Correo electrónico:m-grauto@uniandes.edu.co

**** Economista, magíster en Economía, Universidad de los Andes. Investigador, docente Universidad Libre, docente Instituto de Posgrados, Fórum, Universidad de La Sabana, Correo electrónico: fchavarr@uniandino.com

****** Internacionalista y politóloga. Universidad Militar Nueva Granada. Correo electrónico: jazminbalaguer@msn.com 


\begin{abstract}
The paper analyzes the public policies of security in the city of Bogotá, based on the diagnosis that there is a correlation between crime and the objective causes of the conflict. However, the empirical evidence of the literature review, shows that this relationship was not significant, which allow us to develop a model of criminal behavior, to explain the phenomenon as a competition where the offender is competing with other individuals, forming a structure that improves the productivity of the criminal
\end{abstract}

\section{Introducción}

El presente artículo realiza el estudio de los factores que inciden de forma significativa sobre el crimen de alto impacto en la ciudad de Bogotá; desarrolla un modelo de comportamiento delictivo frente a incentivos y costos económicos. El postulado de que el delincuente es fruto de la genética, disgregación familiar u otras condiciones psicosociales fue desvirtuado a partir de la teoría racional del crimen y su posterior confirmación por intermedio de evidencia empírica. En Colombia la literatura denominada "sociología de la violencia", es decir, investigación que se elabora a partir de la crónica periodística y entrevistas con los actores y testigos de hechos violentos, reafirma la tesis de la inclinación genética al crimen por parte de la sociedad; esto indica como postulado que Colombia es proclive a la actividad violenta y criminal.

Este axioma genera la sabiduría convencional que predomina en la elaboración de políticas de seguridad focalizadas sobre el comportamiento psicosocial, desconociendo la fuerza que tienen los incentivos sobre el comportamiento de las personas. Según Levitt (2006, pp. 23-24) chain, eliminating less fit agents in criminal activities. The empirical evidence is preliminary because there is no access to micro-data from the victimization survey in 2003, which have been declared reserved by the Colombian government.

Key words: Crime, Broken Windows, Theory of Crime Wise, tournament theory, risk aversion.

Glassification JEL: A12, B21, G61, G73.

el sistema de incentivos se cuadruplicó desde que Adam Smith escribiera "La riqueza de las naciones" en el siglo XVIII; por lo tanto las ciencias sociales deben usar el instrumental técnico que poseen, a fin de afrontar temas que convencionalmente son abordados por expertos en justicia, defensa y seguridad. Las valoraciones morales desencadenan un comportamiento maximizado de felicidad que minimiza el dolor (Bentham, 1982).

A partir de la solución descentralizada del primer teorema del bienestar, Becker planteo un modelo racional que explica la conducta criminal, entendiendo racionalidad como la preferencia por poseer más sin llegar a alcanzar los límites de saturación. Los investigadores han focalizado su atención en contrastar la teoría racional del crimen a través de la evidencia empírica que permite dar validez a dicha tesis (Becker, 1968, pp.169-217).

Becker al recibir el premio Nobel en 1992 dio un ceremonial discurso, donde explicó el surgimiento de la teoría; esta floreció cuando tenía que atender un examen oral y realizó una valoración de dejar mal estacionado su automóvil, con los riegos que implicaba ser multado, o llegar 
a tiempo y cumplir sus actividades académicas dejando intacta su reputación. La solución del dilema es igual, al beneficio de llegar a tiempo conservando su buen nombre, menos la probabilidad de ser atrapado ejecutando una infracción de tránsito; como el riesgo de ser sorprendido era bajo, (Becker, 1992) el profesor Becker cometió el acto delictivo llevándolo a un equilibrio de Nash que concluye: "Lo bueno para sí mismo no es bueno para la sociedad" (Nash, 1950, pp. 48-49).

Los individuos optan por cometer infracciones con base a costos y beneficios de sus acciones; detrás de esta lógica se oculta la forma de valorar los incentivos que generan la política pública de seguridad. Los costos son las acciones de seguridad que elevan el precio de cometer un delito; como lo es la probabilidad del arresto, tasa de condena, niveles de vigilancia, multas pecuniarias y condenas a la pena capital. Trabajos más recientes han derivado en mediciones de las políticas de legalización del aborto, control de armas y prohibición del consumo de drogas. La revisión bibliográfica encontró que la literatura científica que se ocupa del tema de la criminalidad en la ciudad de Bogotá es escasa, pese a que la ciudadanía ha revelado que el problema de la inseguridad es una de las principales preocupaciones ${ }^{1}$. Este trabajo aporta al entendimiento del fenómeno criminal de Bogotá, haciendo énfasis en que el estudio de esta problemática se abandonó a favor de las investigaciones del conflicto armado; además, la política pública percibe la actividad delictiva como la consecuencia de una problemática social.

1 Tomado de: Encuesta de Percepción y Victimización en Bogotá y las localidades; "El 39\% de las personas encuestadas consideran que la inseguridad ha aumentado en Bogotá. Se ubica por debajo del promedio de los 11 años de aplicación (44\%). Se mantiene esta sensación respecto a diciembre del año anterior, pero aumenta en 5 puntos frente a junio de 2007, Vicepresidencia de Gestión Cívica y Social, Dirección de Seguridad y Convivencia.
El documento se encuentra organizado de la siguiente forma: en la primera parte se halla la presente introducción, en la segunda sección se realiza la revisión de la literatura que ha dado aportes relevantes; el tercer apartado analiza la política pública de seguridad de Bogotá; la cuarta parte desarrolla un modelo sobre comportamiento criminal basado en la Teoría del torneo; la quinta parte presenta evidencia empírica tomada de la encuesta de victimización del año 2003; al final se presentan las conclusiones

\section{La política pública de seguridad de Bogotá D.C.}

La Seguridad es un bien público que debe ser proveído por el Estado a través de políticas oportunas, eficientes y sostenibles. En Bogotá, la política pública de seguridad se desarrolla desde la Administración Mockus (1995) y Peñalosa (1998). Antes de este período las políticas públicas en seguridad fueron escasas; durante el siglo XIX Bogotá no contaba con recursos suficientes para darle atención a las primeras migraciones agrarias que no poseían redes de alcantarillado. Pese a las limitaciones fiscales de la ciudad, se construyó el tranvía, el acueducto, el alumbrado público a gas y eléctrico, y el servicio telefónico; asímismo se impulsó el desarrollo de espacios urbanísticos como el Parque Centenario y un proceso de industrialización, que se evidencia en la creación de empresas como Cementos Samper (1909) y Bavaria (1889).

El comercio se asentó en el centro de la ciudad, lo que produjo que familias con altos ingresos se trasladaran a zonas periféricas. La presión demográfica fue el principal problema que afrontó Bogotá a comienzos del siglo XX. Hubo en principio incapacidad de albergar y dotar a esta población de infraestructura, pero se contrarrestó con la creación del Departamento de Urbanismo del municipio 
de Bogotá (1893), que inició la institucionalidad que daría soluciones a los problemas urbanísticos de la capital.

El gobierno de López (1934-1938), así como el gobierno local de Gaitán, desarrollaron obras arquitectónicas de gran envergadura como las Avenidas Chile y Caracas, así como la planificación de los barrios Palermo, Campín, construcciones de edificios residenciales, autopistas, centros culturales y hospitales.

El interés de la ciudad por continuar con obras arquitectónicas, se evidencia en las políticas urbanas del alcalde Mazuera (1948-1949), el cual realizó el primer plan de desarrollo de Bogotá, se propuso conservar sólo una parte del centro histórico de la ciudad, construir modernas edificaciones alrededor de la Plaza de Bolívar y la carrera séptima; dichas iniciativas no serían acogidas pero sí lo fueron unas disposiciones que permitieron regular servicios públicos y la urbanización hasta ese momento.

Esa modernización se alteró con el asesinato del candidato presidencial Jorge Eliécer Gaitán (1948); este hecho desencadenaría los disturbios y destrucción del centro de la ciudad. Por ello las políticas de la ciudad hasta finales de los años cuarenta estuvieron orientadas a la reconstrucción de la ciudad.

Las políticas públicas en seguridad incluso no se habían planteado, en primera instancia porque hasta 1954 la ciudad obtuvo la condición de Distrito Especial, lo que permitió autonomía administrativa, separándola administrativamente del Departamento de Cundinamarca. Sólo hasta ese período Bogotá obtuvo recursos fiscales propios, lo que haría posible diseñar y formular políticas de acuerdo a los requerimientos de la ciudad, pero las condiciones de inseguridad del momento no hacía necesario la creación de políticas de seguridad.
A mediados de la década del cincuenta inicia una masiva migración campo-ciudad impulsada por las mejores oportunidades laborales y condiciones de vida en Bogotá, sumado esto a la polarización política de este período, llevó a que la ciudad tuvieran 25 alcaldes en menos de quince años lo que provocó la paralización de obras públicas y un crecimiento desordenado de la ciudad. Como resultado de esta situación la vivienda fue precaria para los nuevos habitantes, trayendo a la vez el nacimiento de los cordones de miseria de Bogotá, la capital empezaría a crecer sin planificación, dando como resultando el deterioro de la infraestructura que se había alcanzado, disminución de la calidad de vida y desintegración del tejido social, la desigualdad social surgida bajo estas condiciones hará estragos en el futuro de la seguridad ciudadana.

Bogotá para entonces afrontaba la más alta tasa de crecimiento demográfico $(7 \%)$, la administración Virgilio Barco (1966-1969) enfrentó esa problemática, no obstante, las políticas distritales estaban focalizadas a espacios públicos, sistemas de parques, vivienda, vías y transporte masivo, así como en dar cobertura de vivienda y transporte; el tema de seguridad no se contemplaba en la agenda de Gobierno (Gerard \& Ceballos, 2004).

Entrada la década del ochenta, el país en general inicia la escalada criminal con repercusiones nefastas gracias a las actividades de organizaciones delictivas y criminales tales como narcotráfico y grupos armados ilegales, que empeoraban cada vez más con la evidente ineficiencia política de la cual Bogotá no fue la excepción, pero que ante la eminente situación de criminalidad e inseguridad generalizada se empiezan a formular las primeras políticas en seguridad.

El presidente Virgilio Barco (1986-1990), concibe políticas que ya no corresponden a una modernización de su infraestructura, ahora la focaliza sobre los problemas que 
acechan la convivencia social y la seguridad del Estado. Por tal razón, y por primera vez se plantea una política pública de seguridad ${ }^{2}$, aunque no de índole local sino de carácter nacional, que consistía principalmente en la tipificación de las conductas delictivas y terroristas, modernización de la administración de justicia; la tasa de homicidios aumentaba a un valor máximo histórico.

El Alcalde Julio Cesar Sánchez (1986-1988) (Casas \& González, 2005) crea los centros de atención inmediata (CAI), que estarían a cargo de la política de la ciudad, como un servicio comunal publico de información y respuesta oportuna que permitiría dar protección a los habitantes en situaciones de violencia instrumental. Este fue un avance en formulación de políticas públicas dirigido a salvaguardar la seguridad de los habitantes locales.

A finales de la década del ochenta y comienzos de los noventa, no existía una política pública clara, con delimitación de responsabilidades en todas las escalas de la administración pública, que respondiera a las necesidades de seguridad de la ciudad de Bogotá. La seguridad era vista como un tema de índole nacional y con actores claros de desestabilización de alcance nacional como los grupos insurgentes. No obstante, el presidente César Gaviria (1990-1994), tuvo una visión más amplia de la violencia del país, al entender que las ciudades necesitan una política de seguridad orientada a la población, esta es la primera vez que se establece la seguridad ciudadana como una política integral aunque sea de orden nacional. Esta política de seguridad nacional se denominó: "Estrategia nacional contra la violencia", que se caracterizó por la inserción en la sociedad civil de los grupos guerrilleros emergentes tales como el EPL, M-19 y el Quintín Lame. Así como la confrontación contundente contra los carteles de droga ilícitas.

2 Decreto 180 de 1988.
La represión del gobierno de César Gaviria hacia los carteles de las drogas ilícitas, hace que Bogotá, que padecía la persecución del cartel de Medellín contra los miembros de la justicia y la Policía Nacional de forma sistemática, a través de atentados y asesinatos indiscriminados. En 1991 alcanzó los 80 homicidios por 100 mil habitantes (hpcmh), el más alto durante toda su historia. A partir de esa fecha, también se aprecia una inflexión y se inicia un descenso generalizado en las tasas de criminalidad, siendo la tasa de homicidios para el año 2003 de 23 por cada 100 mil habitantes (hpcmh) .

El acelerado descenso de dicho indicador en la ciudad de Bogotá es tema de debate en cuanto a cual es la causa de dicho comportamiento. Por un lado existen académicos que afirman que la disminución paralela de la criminalidad en el país fue gracias a la contundente persecución a los carteles de la droga por parte del gobierno de César Gaviria, lo cual alivió, en ciudades como Bogotá, las consecuencias de las confrontaciones entre traficantes de drogas.

De otro lado está quienes afirman que la recuperación sostenida de los índices de criminalidad de la ciudad para mediados de los años noventa, se debe a las exitosas administraciones locales de Antanas Mockus (1995-1998), Enrique Peñalosa (1998-2001) Antanas Mockus (20012004). En 1999 la tasa de homicidios retornó a los 25 por 100 mil habitantes, que es el promedio histórico anterior a los años ochenta. Sin embargo, la tasa nacional también convergió a su promedio histórico, aunque sigue siendo un poco más alta, por efecto del escalamiento del conflicto durante estos últimos años ${ }^{4}$.

3 Datos de la Policía Nacional y el Ministerio de Defensa para el año 2007

4 Serie Nacional: Base de datos Policía Nacional, Centro de Investigaciones Criminológicas, 2005. 
En el año 2000, el mayor número de víctimas en la ciudad no estuvieron relacionados con la confrontación armada en contra del Estado, sino que son manifestaciones de violencia relacionada directamente a organizaciones criminales locales, y en menor proporción, a la intolerancia social, riña o ajuste de cuentas, saliéndose de las consecuencias de procesos y políticas nacionales en seguridad, anteriores a la administración de Antanas Mockus (1995-1998).

El punto de debate es que el descenso de la criminalidad en todo el país inicia tres años antes de la primera de estas administraciones. El éxito de la administración de Antanas Mockus se debe a que por primera vez un gobierno local diseña políticas públicas en materia de seguridad ciudadana, al dar prioridad a la violencia particular y tipificada al interior de la ciudad, donde se presta atención a la intolerancia urbana. El plan de gobierno se orienta hacia la cultura ciudadana, con el propósito de combatir la violencia en la ciudad:

La cultura ciudadana establece un marco que regula los comportamientos de los ciudadanos, en la medida que establece reglas mínimas comunes que haces posible las relaciones entre sí y con su entorno, donde su principal defensa es para la vida (Acero et ál., 1997).

Otras de las políticas para disminuir los índices de violencia en la ciudad por parte de la administración de Antanas Mockus, es la recuperación del espacio público, así como el desarme ciudadano y la regulación del consumo de alcohol. Para la ciudad esta política es oportuna e independiente de la Política Nacional de Seguridad, atendiendo a los requerimientos y problemáticas de Bogotá; con ella se fortalece la Policía Metropolitana y se reconoce la importancia del acercamiento entre ciudadanos y la Policía comunitaria.
En 1998 se establece la Subsecretaria de Convivencia y Seguridad Ciudadana, como responsable del desarrollo de los planes y programa del gobierno local, dando un paso importante hacia la institucionalidad y modernización al tratar el tema de seguridad en Bogotá. Esto se acompañó durante todo la administración por una fuerte pedagogía orientada a los ciudadanos, con el fin de mejorar su capacidad de comunicación para la solución de conflictos.

El gobierno de Enrique Peñalosa (1998-2000) siguió algunos aspectos de las políticas innovadoras de su antecesor, aunque su prioridad era construir una ciudad moderna y amable, recuperar el espacio público; para generar orden y estética a la urbe, que basa sus políticas en la teoría de las de ventanas rotas ${ }^{5}$. En el 2000 se dio origen a los Consejos Locales de Seguridad, donde se debatía junto con las autoridades civiles y de policía, las necesidades específicas en seguridad, de esta forma se implementaron programas eficientes para combatir los índices de inseguridad de acuerdo a las necesidades por cada localidad.

Uno de los programas exitosos que enmarco la Política Pública en Seguridad fue la de "Zonas Seguras", cuyo objetivo fue garantizar la presencia de policía en aquellas zonas de mayor influencia criminal, dotándolas de unidades móviles y atención especial. Asimismo, el programa de "Misión Bogotá", que poseía la concepción de seguridad como una responsabilidad individual, empleando la vigilancia comunitaria y la convivencia social. Enrique Peñalosa incluyó un lema de seguridad, bajo la premisa

5 Teoría propuesta por los criminólogos estadounidenses Wilson y Kelling hace dos décadas, pone de relieve el impacto del desorden social y del entorno en las percepciones y condiciones de seguridad de las comunidades. Se plantea que la presencia de ciertas características del entorno físico y social, agrupadas bajo el término "desorden" (como basuras en las calles, sitios abandonados, vandalismo, riñas y escándalos callejeros, presencia indigente y prostitutas) emiten señales de decaimiento del orden comunitario que encienden los temores de los habitantes creando un efecto en cadena. 
de: "Prevenir y sancionar los comportamientos que lesionan el bienestar de los ciudadanos e impulsar aquellos que promueven la paz, la solidaridad, el respeto y la vida en comunidad" (Peñalosa, 2000).

En la segunda administración de Antanas Mockus (20012004), a través de su plan de desarrollo "Bogotá para vivir todos del mismo lado", se siguió por la línea de la cultura ciudadana y la defensa del espacio público para disminuir los índices de violencia, bajo el lema "La vida es Sagrada". Se destinaron importantes recursos para el fortalecimiento de la investigación criminal y prevención contra la violencia intrafamiliar y el maltrato infantil.

El alcalde Luis Eduardo Garzón (2004-2007) generó incertidumbre entre la ciudadanía en cuanto, a cómo iba a tratar los temas de seguridad y la política pública en este ámbito en la capital del país. La visión fue pragmática, dejando de lado la cultura ciudadana como principal argumento para combatir la criminalidad en la ciudad. El argumento era que Bogotá tenía múltiples causas que originan violencia urbana y que no se puede dar atención a la demanda de seguridad desde un solo ámbito como el cultural. De esta forma su política de seguridad se centró en la premisa de la multi-causalidad y atención integral a la ciudadanía, especialmente a la población vulnerable; de esta forma, se atacarían problemas que constituyen la inseguridad como la pobreza y la desigualdad, además de rescatar la inclusión social.

Esta política de seguridad da continuidad a las políticas de sus predecesores, teniendo en común la comunicación activa con la ciudadanía, lo que Garzón llamó "participación ciudadana". Esta administración en el 2006 tuvo mucho éxito, reflejado en el descenso de la tasa de homicidios que paso a 18 homicidios por 100 mil habitantes, a la más baja alcanzada en los últimos 21 años, aunque es una de las más altas del país.
Durante la presente alcaldía a cargo de Samuel Moreno (2008-2009), la política de seguridad implementada se caracteriza por la continuación en el acercamiento de ciudadanía con autoridades locales y policiales, el aumento del pie de fuerza que permita la presencia y atención en zonas vulnerables y los consejos comunales en las localidades. No obstante, ante los esfuerzos del alcalde Samuel Moreno, los índices de inseguridad según cifras oficiales se han disparado en la ciudad, aumentando los homicidios de 18\% a 19.2\% del año 2007-2008 (Policía metropolitana). La situación de inseguridad en la ciudad ha empeorado, lo que se evidencia a través de las cifras de victimización y percepción de la ciudadanía. El fenómeno criminal es desconocido para esta administración, lo que lleva a la mala focalización de recursos al implementar políticas que ya se han agotado y que requieren otro tipo de respuesta

\section{Investigación sobre el crimen de alto impacto}

La presente revisión muestra las investigaciones, trabajos y artículos académicos que han tenido impacto en el desarrollo de políticas públicas de seguridad, que por su contenido han generado desarrollos teóricos que permiten entender los fenómenos delictivos o toman como objeto de estudio la criminalidad de la ciudad de Bogotá. Este marco conceptual permite a la investigación avanzar sobre nuevos elementos del fenómeno delictivo, ahondar en vacíos conceptuales que dejaron dichas investigaciones, y establecer un diálogo académico sobre temas que generan divergencias debido a la metodología que se empleó e hipótesis formuladas.

Las principales investigaciones nacen del Centro Paz Pública de la Universidad de los Andes, su producción intelectual cesa a partir de 2005, lo que genera una caja 
negra, la continuidad de las investigaciones se pierde; como consecuencia la producción de políticas públicas presentan sesgos por parte de quienes las elaboran, un claro ejemplo de ello es: "Proyecto de Acuerdo: Por el cual se reviste al Alcalde Mayor de Bogotá, de facultades extraordinarias para aumentar el pie de fuerza policial en el Distrito Capital y se dictan otras disposiciones".

En dicho proyecto resaltan los juicios de valor del ponente $^{6}$, la exposición de motivos no presenta las citas bibliográficas de donde obtuvo la información; el desconocimiento del carácter misional y nacional de la Policía Nacional de Colombia es latente. La ignorancia por parte del legislador es consecuencia de la ausencia de investigación científica en el campo de seguridad.

\subsection{Investigaciones realizadas}

La revisión del objeto de estudio da como resultado que doce documentos se concentran en el delito de alto impacto de la ciudad. La descripción que se presenta a continuación, tiene como objetivo mostrar las conclusiones a la que llegaron los autores, la metodología empleada y su limitación. Finalmente, mostrar los vacíos que dejaron las investigaciones; el propósito es generar un diálogo académico.

El primer trabajo relevante que aborda el tema de la criminalidad es Rubio $^{7}$ (1998), este documento emplea

6 Ver Parada, O. (2007). “ ...por el cual se reviste al Alcalde Mayor de Bogotá, de facultades extraordinarias para aumentar el pie de fuerza policial en el Distrito Capital y se dictan otras disposiciones"; Proyecto de Acuerdo No. 425 de 2007, Concejo de Bogotá D.C.

7 Ver Rubio, M. (1998). "Criminalidad urbana en Colombia", Centro de Estudios sobre Desarrollo Económico, Facultad de Economía; Paz Pública, Programa de Estudios sobre Paz, Justicia y Violencia, Universidad de los Andes. la Encuesta Nacional de Hogares en el módulo de criminalidad y victimización. A partir de la información plasmada en dicha herramienta, analiza el set de datos con métodos estadísticos y concluye con el siguiente argumento; las causas objetivas de la violencia que han dado el argumento central a la teoría del derecho penal, no explican la acción criminal. El autor es contundente al afirmar que: "Las peculiaridades colombianas son también el resultado, y no simplemente la causa de una situación de violencia desbordada".

$\mathrm{Al}$ argumentar que los brotes de violencia, no controlada oportunamente, evolucionan en escenarios de crimen organizado, que desbordan la capacidad de control de las autoridades encargadas de la seguridad. Los resultados que expone la investigación ${ }^{8}$, es que la criminalidad se encuentra dentro del promedio internacional; pero con una singularidad, poseer una inclinación a emplear violencia desproporcionada contra las víctimas de atentados a la propiedad. Asimismo, si los ofendidos son jóvenes la probabilidad de denuncia es menor; de esta forma los infractores evalúan los costos de emprender acciones criminales.

La investigación igualmente concluye que existe una relación negativa entre delitos que se denuncian e incidencia de atentados contra la propiedad lo que comprueba una de las principales predicciones de la teoría económica del crimen, que la mayor propensión a denunciar hechos delictivos, los costos de delinquir se incrementan, generándose una correlación negativa entre atentados contra

8 El 40\% de los delitos económicos se cometen de manera violenta y en las distintas ciudades la proporción nunca es inferior al 25\% y alcanza a ser cercana al 50\%. Por el contrario, en la ciudad con la mayor ocurrencia de muertes violentas se dan, simultáneamente, índices muy bajos de lesiones personales. La letalidad de los atentados contra la vida muestra importantes variaciones regionales alrededor del promedio nacional del 34\% (un incidente mortal por cada tres atentados) que van desde cerca del $70 \%$ hasta menos del $10 \%$. 
la propiedad. Además se encuentra que las ciudades con bajos niveles delictivos presentan niveles más bajos de litigios en áreas no penales del derecho; las ciudades con mayores índices criminales presentan valores superiores al promedio nacional con respecto a otros tipos de conflictos jurídicos.

Un alto índice de denuncias elimina factores criminales y desmiente la sabiduría convencional que afirma que la acción penal no disuade al crimen; por el contrario Rubio (1998) determina que el delincuente, al cometer un acto criminal, se asegura que la víctima no tome acciones contra él, por ello recurre a la violencia fáctica. El temor a que ofendidos tomen acciones penales contra los ofensores dispara la tasa de homicidios. Además, la ciudadanía revela una demanda de seguridad a favor de las autoridades judiciales versus los organismos de seguridad.

Finalmente, el autor rompe el paradigma de los factores psicosociales, tejido social y políticas comunitarias como explicación de fenómenos delictivos. Para ello se recurre a un tecnicismo econométrico denominado autocorrelación, este elemento determina cómo una variable se explica sobre sí misma a través del tiempo, esto quiere decir que la criminalidad de hoy es explicada por la actividad criminal de ayer; si los factores objetivos de la violencia fueran una realidad, el paso del tiempo no debería alterar la tasa de homicidios por cien mil habitantes, es decir, debería presentar tasas que fluctuaran alrededor de una medida. Este no es el caso, el tiempo afecta a dicho índice que los factores descritos no explican el comportamiento criminal.

El artículo de Llorente y otros: "Violencia homicida y estructuras criminales en Bogotá", ataca los lugares comunes que intentan explicar la criminalidad de Colombia, en él se refieren a tres mitos que han pasado a formar parte del ideario que permite dar sentido al fenómeno delictivo: "el primer mito de la violencia cotidiana surge de la intolerancia ciudadana".

Según la evidencia empírica que se analiza concluye que la violencia, producto de la intolerancia en Bogotá, "no sobrepasa el 30\% del total de homicidios". Se indica que el $70 \%$ de las muertes violentas de la capital colombiana corresponden a criminalidad instrumental. Igualmente, los autores emplearon herramientas de probabilidad estadística que permitió inferir que los datos de homicidio en Bogotá (1997-1999) no se distribuyeron de forma aleatoria a través de la ciudad. Lo que apreciaron es que existe una tendencia a concentrar los homicidios en ciertos lugares de la ciudad; además dicho patrón es constante en el tiempo. Con la evidencia que arrojo la muestra de datos se desmintió el hecho de que la violencia es producto de la agresividad colombiana. Asimismo llegan los investigadores a la conjetura que las muertes violentas causadas por riñas y consumo de alcohol se producen en gran parte en lugares conflictivos, concluyendo que no es la presencia de la intolerancia ciudadana la causa de la violencia homicida, "sino del tipo de personas que frecuentan estos escenarios"

El segundo mito ataca el trabajo, la violencia impulsiva escala hacia el crimen organizado e instrumental, para rebatir esta tesis convencional se valen de la correlación que existe entre la violencia instrumental y la que es fruto de la intolerancia y hallan la existencia de correlación positiva del $94 \%$ entre los dos tipos de violencia, además encuentran que localidades donde la tasa de homicidios por violencia instrumental es alta; es donde se encuentra el mayor número de casos de violencia por intolerancia; así descubren una razón de causalidad que sigue la dirección: crimen organizado igual a violencia impulsiva. 
El trabajo de Rubio (1999) a fin de desmentir las causas objetivas como explicación de la violencia homicida, destaca la baja relación entre muertes violentas, número de habitantes y crecimiento poblacional en las localidades más violentas de Bogotá en menor número. Los sectores con un crecimiento demográfico mayor presentan una tasa de homicidios por debajo de la media de Bogotá. Según lo analizado en este apartado, los flujos migratorios y población desplazada no son el origen de la violencia ciudadana.

El siguiente documento: “¿Garrote o zanahoria? Factores asociados a la disminución de la violencia homicida y el crimen en Bogotá, 1993-2002”, genera impacto dentro de la investigación que toma por objeto de estudio el comportamiento criminal de la ciudad de Bogotá, fue elaborado por Sánchez y otros (2003). Este se enfoca en la evidencia empírica y emplea modelos de datos panel con una variante, la aplicación de la matriz de contigüidad espacial; el objetivo es capturar los efectos que genera la distribución de los datos a través de un espacio geográfico. Este método determina el grado de autocorrelación espacial, lo que permite inferir que los hechos delictivos, no sólo dependen de lo que sucede en dicho lugar, sino de acciones delictivas en otras zonas.

Inicialmente, el trabajo describe la historia de las políticas de seguridad de la ciudad entre 1993-2002, luego analiza los datos con estadística descriptiva; posteriormente describe la teoría racional del crimen y la de ventanas rotas; a partir de dicho marco teórico se crea una clasificación de variables que denomina medidas zanahoria, garrote, y ventanas rotas. Con dicha clasificación diseñan un modelo data panel y un modelo espacial panel.

La investigación confirma que las causas objetivas no explican el comportamiento criminal; además infiere que existe un fenómeno de contagio criminal, lo que lleva a concluir la existencia de una criminalidad organizada, la cual se comporta como un vector de contagio; quiere decir que las zonas violentas dispersan el crimen a sus alrededores. La tercera conclusión es que la tasa de capturas explica: " $53 \%$ de la disminución en la tasa de homicidios y el $76 \%$ del cambio en la tasa de atracos".

$\mathrm{Al}$ elevar los costos de la actividad criminal, trae como consecuencia drásticas reducciones de los índices delictivos, confirmando la predicción de la teoría racional del crimen. El trabajo se aproxima a responder, qué causas provocaron el descenso de los índices delictivos desde 1993; presenta una falla metodológica, los autores no contrastan la evidencia empírica con lo sucedido antes del punto de inflexión, lo que deja el interrogante: ¿por qué ascendió la criminalidad en la década de 1980?

Si las mismas políticas de contención delictivas se aplicaron en la década de 1980, habrían generado el mismo impacto, o existieron índices similares en lo que respeta a nivel de capturas. Preguntas de este tipo no son posibles a causa de esta falla metodológica. Igualmente, no se comprueba la existencia de un cambio estructural del comportamiento criminal; o si es la respuesta de seguridad la que determinó la caída del crimen de alto impacto.

En el escrito "Homicidio e intención letal: un estudio exploratorio de heridas mortales a partir de los protocolos de necropsia en Bogotá" elaborado por De León y otros (2003), permite dar mayores elementos empíricos que corroboran las tesis: el delito de alto impacto de la capital, es ejecutado por personas expertas en el manejo de armas de fuego. Los autores llegan a inferir que existe: "un fenómeno de profesionalización delictiva".

Para llegar a estas conjeturas se toman las estadísticas que existen sobre las armas que generaron la muerte violenta. Esta metodología resulta novedosa por que en lugar de tomar bases de datos del número de homicidios, toman el 
elemento empleado para cometer el hecho delictivo. Esta metodología permite estudiar de manera indirecta el patrón de comportamiento del victimario, en la exposición de motivos aducen, que no se podría extraer una muestra aleatoria de los homicidas en un centro de reclusión debido a que en dichas instituciones permanecen personas que por su inexperiencia fueron capturadas, este argumento se sustenta a continuación:

Sólo 7\% de los homicidios llegan hasta la etapa de sentencia condenatoria. En una revisión de investigaciones por homicidios que alcanzan dicha etapa muestran que los homicidios que resuelve el sistema penal son los homicidios triviales, es decir, aquellos que se cometen delante de muchos testigos y por lo general relacionados con el licor. (De León et ál., 2003).

Las estadísticas que analizan los autores (De León et ál., 2003), muestran que las heridas con arma de fuego se producen en un $55 \%$ de las veces en la cabeza y con arma blanca en un $80 \%$ de los casos en el tórax, partes letales de la anatomía humana. Bajo condiciones de tensión, propinar disparos o golpes certeros con instrumentos cortantes es una acción que requiere pericia y control emocional, se concluye que las personas que ejecutan dichas acciones han recibido una preparación técnica para cometer este tipo de actos. Así se establece que la delincuencia bogotana está preparada en un ambiente criminal y profesional, no en un ámbito de supervivencia social.

Ser delincuente bajo este escenario requiriere desarrollar destrezas, adquirir conocimientos delictivos y elaborar métodos para cometer acciones criminales; con el propósito de ascender en un ambiente altamente competitivo. Esta conjetura resulta de analizar las cifras que presenta el trabajo de Badel-Trujillo (1998), donde encontraron que el 38\% de las víctimas poseían antecedentes delictivos; lo que implica que eliminar competidores es una alternativa en el ascenso de un criminal profesional.
Bajo esta misma línea, De León presenta un nuevo escrito: "Una descripción de las armas de fuego homicidas en Bogotá para el año 2002 y una propuesta para aumentar el costo del servicio de homicidio" (2004) (continuación del documento previo), donde levanta una muestra sobre el tipo de armas de fuego empleadas para cometer los asesinatos y presenta una acción pragmática con el propósito de elevar el precio de cometer homicidios de tipo instrumental. Las personas que se dedican a esta actividad de forma profesional, valoran el anonimato de sus acciones, por lo tanto: "el valor de sus activos es decir las armas".

Es un recurso valioso si la probabilidad de identificar un arma que participó en un crimen es baja, la cotización de dicho bien se eleva. $\mathrm{Al}$ recurrir a la teoría racional del crimen, que afirma que sí los costos de una acción criminal se eleva, genera la señal para que la demanda por acciones delictivas decrezca de forma sensible. Para el autor de la investigación desarrollar una política de identificación de armas de fuego disuadirá la acción violenta e instrumental.

Los documentos de De León son novedosos en cuanto al objeto de estudio, valiosos en el sentido de comprobar la teoría racional del crimen, al reafirmar que la criminalidad Bogotána posee un grado de experticia alto; que contradice los lugares comunes, como el ejemplo convencional de un par de personas consumiendo alcohol que desencadenan un acto de violencia intolerante con consecuencias fatales. Los documentos tienen inclinación a convertirse en recomendaciones de política pública de seguridad, debido a ese carácter deberían plasmar el cómo instrumentalizar dichas acciones, además las muestras no son aleatorias, si bien los autores reconocen ese hecho, esta implicación resta validez a sus resultados.

El artículo de Moreno: "Impacto de Transmilenio en el crimen de la avenida Caracas y sus vecindades" (2005), estudia el fenómeno delictivo de Bogotá al tomar como 
experimento natural la instauración del sistema de Transmilenio en la ciudad. El sistema de transporte masivo permite eliminar los problemas de endogeneidad que presentan los estudios criminológicos; el autor recurre a documentos que han estudiado ataques terroristas en otras ciudades (Di Tella \& Schargrodsky, 2004), y que han permitido aislar el fenómeno delictivo y extrapolar conclusiones que tienen en cuenta las razones de causalidad del mismo.

Según el autor, el recorrido del sistema de transporte masivo a través de la Avenida Caracas, permite que una distribución aleatoria en el espacio geográfico propicie una presencia delictiva de alto impacto. Por lo tanto, las medidas de seguridad que se han aplicado en la troncal de la Caracas, no presentan problemas de simultaneidad. El método empírico que empleó el autor fue la econometría espacial, esto con el propósito de capturar los fenómenos de contagio y desplazamiento criminal; de igual forma recurrió a la metodología costo-beneficio para evaluar el impacto que tuvo el fenómeno delictivo con respecto al nuevo sistema de transporte de Bogotá.

Los resultados a los que llegó la investigación muestran una caída de los índices delictivos, posteriores a la instauración del sistema de transporte, igualmente determina la existencia de autocorrelación espacial que permite inferir que cierta zonas geográficas de la ciudad son conglomerados de actividad criminal, donde las economías de escala permiten que el mercado delictivo sea eficiente. Se destaca que en el sur de la ciudad el nivel delictivo descendió de forma significativa, mientras que el norte de Bogotá sufre un efecto de desplazamiento criminal.

Este documento presenta una novedosa metodología de evaluar el fenómeno delictivo, llega a resultados robustos econométricamente, pero algunas interpretaciones de los ejercicios empíricos no son claros, y dejaron vacíos en al- gunos resultados que no se interpretaron en dicho experimento natural. El documento analiza cuatro años, esto no permite validar los resultados que arroja.

La siguiente investigación: “¿Más Crimen?, ¿más Miedo?", desarrollo y sociedad, primer semestre 2007" (Restrepo, 2007), es una medición del miedo a ser victimizado en la ciudad de Bogotá, es el primer documento que emplea métodos estadísticos para valorar el miedo, generalmente se han elaborado encuestas sobre percepción, que su máximo avance es hacer descripciones estadísticas.

La autora empleó una muestra de 3400 personas de la Universidad de los Andes, que respondieron a una encuesta sobre victimización y medidas que toman frente a fenómenos delictivos, independientemente de si fueron o no víctimas de actos criminales. Los resultados mostraron que la victimización es inferior a las medidas de autoprotección que toman las personas, es decir, el nivel de miedo es mayor que la probabilidad de ser víctimas de un hecho delictivo, esto confirmó la evidencia que arrojan otros estudios sobre la medición del miedo en otros países.

Igualmente, se muestra que el nivel de miedo se refleja en las medidas de seguridad que se toman, pero esto trae consigo una paradoja, las medidas de autoprotección refuerzan el círculo del miedo, esto quiere decir que a mayores medidas, mayor es el nivel del miedo. El documento confirma que la edad y el sexo son variables que tiene en cuenta el delincuente para iniciar su acción sobre una persona (víctima), así, la teoría racional del crimen desmiente que los medios de comunicación sean un canal de transmisión que catapulte los niveles de percepción de inseguridad.

El estudio presenta modelos probit que miden el grado de temor de la ciudadanía, pero la investigación presenta una deficiencia y es la forma como se extrajo la muestra. 
En primer lugar sólo toma a la comunidad de Los Andes como el objeto de estudio y en segundo la encuesta se distribuyó a través de los correos electrónicos. Esto rompe el principio que la muestra se debe extraer aleatoriamente, además, al tomar la encuesta vía electrónica, genera sesgos en la forma en que se responde a las preguntas. Así el estudio mide el miedo de una población, pero no se puede inferir que éste sea válido para el resto de la ciudad de Bogotá.

El documento de Gaviria et ál:: "The Cost of Avoiding crime: the case of Bogotá" (2008), es seminal en varios enfoques, en primer término calcula los costos que implica para la población bogotana autoprotegerse del crimen. En este punto recurren al método de precios hedónicos, que consiste en valorar las viviendas en función del número de habitaciones, baños, cercanía a lugares que desmejoran la calidad de vida; y variables que denominan de armonía social, como son la estratificación catastral, provisión de servicios públicos, proximidad a terminales aéreos, parques y estaciones de policía. La variable dependiente es el logaritmo natural del precio de la residencia, éste se calcula preguntando al dueño el valor que estaría dispuesto a pagar por la vivienda si fuera en arriendo.

Posteriormente, se valen de la econometría espacial con el propósito de aislar los efectos de autocorrelación que exhiben los datos de la encuesta de calidad vida, estos se encuentran distribuidos en un área geográfica que genera problemas con las estimaciones de los parámetros, si emplean enfoques clásicos de la econometría. Este método se utiliza con frecuencia en análisis criminológicos, lo novedoso de la investigación es que los autores recurrieron dentro de un modelo espacial al empleo de variables instrumentales, las cuales deben poseer dos características, explicar las variables independientes a instrumentalizar mas no correlacionarse con la endógena.
Los autores emplean las variables: madres adolescentes y diferencias de edad entre hijo y madre, afin de instrumentalizar la variable, tasa de homicidios; estas presentan las características descritas anteriormente, no correlacionadas con los precios de la vivienda, pero sí con el nivel de actividad criminal (Donohue, 2000). Este argumento rompe esquemas con trabajos anteriores, infiere que la tasa de natalidad en un ambiente de mercados criminales presiona de forma positiva los delitos de alto impacto. Adicionalmente muestra cómo la actividad delictiva deriva en mercados de autoprotección que revelan que la provisión del bien público, la seguridad es insuficiente, así el mercado de la seguridad sea incompleto y genere exclusión con los hogares pobres. De esta forma los hogares se valorizan positivamente en presencia de medidas de vigilancia pasiva o activa y estaciones de policía.

La investigación encuentra correlación positiva entre la tasa de homicidios y madre adolescente; asimismo, descubre relaciones espaciales del crimen de alto impacto, en el suroriente y nororiente de la ciudad, con respecto al fenómeno de embarazos prematuros. Este documento abre un espectro de preguntas con la relación existente entre demografía y actividad criminal de Bogotá.

\section{El modelo}

La criminalidad es una actividad racional sujeta a un factor de aversión al riesgo, tal como lo describiera Becker (1968), los delincuentes son agentes que valoran los beneficios y costos de cometer una acción. Según las estimaciones que estos realizan, cometen diferentes tipos de delitos, lo que lleva a considerar que existe un mercado de actividades delictivas. Como todo mecanismo de mercado, posee formas de asignación de recursos y recompensas por ser más productivo en la actividad que desempeña. 
Esta es la causa que permite explicar por qué las condiciones objetivas del crimen han dado resultados no significativos; tal como se describiera en la revisión bibliográfica. Quiere decir esto que la actividad criminal responde a un desarrollo de capacitación, que hace de los delincuentes individuos que maximizan sus recursos, y que disputan con otros el ascenso en la cadena de jerarquía criminal. Desde la teoría del torneo Lazear (1979, pp. 841-864) explica cómo la productividad marginal, relativa de un delincuente, permite el surgimiento de clúster criminales que reducen los costos, maximizan beneficios y atraen personas racionales a este ámbito.

La teoría se desprende del match de tenis donde un jugador avanza en función de su capacidad frente a otro jugador, independiente del nivel de juego de los demás jugadores. Esta forma de competencia permite que los jugadores entreguen su máximo rendimiento gracias a que los incentivos se fijan con anticipación, la sumatoria del comportamiento de todos los jugadores da un rendimiento máximo condicional. Este esquema elimina el riesgo moral de los participantes de este esquema.

El resultado de este sistema se emplea como una solución en la productividad de las firmas, debido a esto el mercado laboral logra mejorar la eficiencia y asignación de recursos, logra que los trabajadores compitan con el objetivo de obtener un beneficio alto. El mecanismo laboral de la criminalidad opera con precisión, en primer término coloca un incentivo muy alto previamente, lo que genera que un número de personas entran a competir por el premio de la actividad delictiva, sea creciente, propicia un esquema de aprendizaje que mejora la capacidad para delinquir, y elimina competidores que no cumplen con las condiciones para permanecer dentro de este ámbito. Esto forma un mercado competitivo que mejora la productividad del clúster criminal, la existencia de grandes líderes delincuenciales, es el incentivo para entrar en la actividad. La eliminación por parte de competidores o autoridades garantiza que se mejoren las estrategias y tácticas del sector delictivo.

La primera ecuación es una función lineal que refleja aversión al riesgo neutral, la escogencia de esta forma funcional tiene como fuente el trabajo de Levitt-Venkatesh (2000), los cuales detectaron que bandas criminales que tienen como fuente de ingresos el crack, presentan este tipo de comportamiento. Un criminal $\{i\}$ obtiene una producción $\{\mathrm{j}\}$ de acuerdo a su habilidad delictiva $(\psi)$, y al componente aleatorio de sobrevivencia y éxito criminal $(\varepsilon)$. De esta forma el mecanismo de incentivos obtiene el máximo valor de la habilidad delincuencial, la distribución que sigue el componente aleatorio es $E[\varepsilon]=\sigma^{2}$ :

$$
q_{i}=\left(\psi_{i}\right)+\left(\varepsilon_{i}\right)
$$

El segundo paso es analizar el valor esperado de remunerar la producción criminal a través de la variable $\{\mathrm{r}\}$ menos los costos de la habilidad delictiva $\mathrm{C}\left(\psi_{i}\right)$, el resultado es la diferencia del beneficio delictivo menos los costos de la habilidad criminal.

$$
E[r q-C(\psi)]=r \psi-C(\psi)
$$

$\mathrm{Al}$ derivar la expresión (2) con respecto a la variable habilidad delictiva $\{\psi\}$, se obtiene:

$$
\mathrm{r}-C^{\prime}(\psi)=0 \Rightarrow C^{\prime}(\psi)=r
$$

Así, el costo marginal iguala al retorno marginal de la habilidad delictiva, lo que traduce en un mercado criminal competitivo que forma un precio $\{\mathrm{V}\}$, el valor esperado del beneficio criminal es:

$$
E[V q-r q]=(V-r) \psi
$$


De esta forma, el costo marginal de la criminalidad es igual a la pérdida social, un modelo que emplea esta estructura se conoce como "destajo", es decir, paga en función de la cantidad de actos delictivos que comete.

$$
C^{\prime}(\psi)=V
$$

Esta estructura no explica la conducta criminal, la aproximación al comportamiento delictivo es la de tipo torneo que presenta una forma funcional donde existen dos agentes criminales, cada uno de ellos representa el numero n de participantes del torneo. En donde compiten por el premio $W_{1}$ el perdedor recibe $W_{2}$, las reglas del juego son determinadas por el orden social establecido. Los delincuentes $j$ y $k$ identifican su producción criminal como una variable independiente que se distribuye de acuerdo a la siguiente expresión: $E\left[\varepsilon_{i}\right]=0$ y $E\left[\varepsilon_{i}\right]=\sigma \square$

El ganador del concurso lo determina la cantidad de actos criminales que generen un alto nivel de producción criminal, el orden del torneo permite clasificar a los participantes del mismo sin que ello afecte los ingresos, el proceso es auto-catalítico, es decir, se replica y mejora sobre la marcha, y al eliminar los individuos menos aptos para la actividad criminal, a la vez que genera altos beneficios monetarios, mejora estrategias y tácticas criminales. Los agentes que participan del torneo conocen las reglas del juego, las estrategias y el premio de forma anticipada, pero no pueden comunicarse, realizar alianzas ni practicar colusión. A través del premio se halla el equilibrio competitivo, por lo tanto, los costos de la actividad delictiva $C(\psi)$ es igual para todos los agentes; así la función de utilidad esperada se expresa de la siguiente forma:

$$
\mathrm{P}\left(\mathrm{W}_{1}-\mathrm{C}(\psi)\right)+(1-\mathrm{P})\left(\mathrm{W}_{2}-\mathrm{C}(\psi)\right.
$$

$\mathrm{Al}$ desarrollar los productos de la expresión, resulta:

$$
\begin{aligned}
& P W_{1}-P C(\psi)+(1-P) W_{2}-(1-P) C(\psi) \Rightarrow P W_{2}- \\
& P C(\psi)+(1-P) W_{2}-C(\psi)+P C(\psi)
\end{aligned}
$$

Simplificando se obtiene la función de utilidad esperada:

$$
P W_{1}+(1-P) W_{2}-C(\Psi)
$$

La probabilidad de que $j$ sea el ganador depende de la probabilidad $p$, la cual se encuentra en función de los costos de aprendizaje criminal y de choques aleatorios que afectan la producción delictiva; si $j$ ha dedicado mayores esfuerzos en el desarrollo de sus habilidades criminales y los choques estocásticos son menores que los de $k$ este será el ganador del premio. Así $\varepsilon=\left(\varepsilon_{k}-\varepsilon_{j}\right)$; donde las ganancias dependen de las destrezas criminales que desarrollan los individuos que participan en un mercado delictivo.

\section{(9) $P=$ probabilidad $\left(q_{j}>q_{k}\right)=$ probabilidad $\left(\psi_{j}>\psi_{k}>\varepsilon_{k}-\varepsilon_{j}\right) \Rightarrow$ $P=\operatorname{probabilidad}\left(\psi_{j}>\psi_{k}>\varepsilon\right)=G\left(\psi_{j}-\psi_{k}\right)$}

La hipótesis Nash-Cournot afirma que cada agente optimiza sus habilidades en función de las destrezas óptimas de su mejor oponente, como no conoce al resto de individuos que participan del mercado criminal, éste maximiza sus habilidades con respecto al mercado. De esta forma el jugador $j$ toma como un dado las habilidades que han desarrollado $k$ y viceversa. Así un individuo es parte del mercado, lleva al teorema, "el todo siempre es mayor que sus partes", así no tiene influencia sobre el sistema, pero sí lleva a mejorarlo al tomar decisiones en función a este raciocinio. Trasladar al lenguaje matemático esta deducción implica desarrollar la primera derivada con respecto a la habilidad criminal en la ecuación (7), posteriormente se determina la segunda derivada, la cual es menor que 
cero, así maximizar la probabilidad de triunfo en función de las destrezas criminales.

(10) $\left(W_{1}-W_{2}\right) \quad\left((\partial P) /\left(\partial \psi_{i}\right)-C^{\prime}\left(\psi_{i}\right)=0 \Rightarrow\left(W_{1}-W_{2}\right)\left(\left(\partial^{2} P\right) /\right.\right.$ $\left(\partial \psi_{i}(\bar{D})-C^{\prime \prime}\left(\psi_{i}\right)<0\right.$

$\mathrm{Al}$ derivar la expresión (9) con respecto a la habilidad criminal se obtiene:

$$
(\partial P) /\left(\partial \psi_{i}\right)=\left((\partial G) /\left(\partial \psi_{i}\right)\right)\left(\psi_{i}-\psi_{k}\right)=g\left(\psi_{i}\right)-\left(\psi_{k}\right)
$$

Esta expresión se sustituye en la ecuación (10), donde se desarrolló la primera derivada y se igualó a cero obteniendo la función de reacción del agente $j$.

$$
C^{\prime}\left(\psi_{-}\{i\}\right)=\left(W_{1}-W_{2}\right) g(0) \quad i=\{j, k\}
$$

Este resultado llega a un equilibrio de Nash, sólo cuando las destrezas de $j$ y $k$ son iguales, para que esta situación se presente es necesaria la existencia de patrones estocásticos que generen alteraciones en el desarrollo del torneo. La criminalidad posee choques exógenos, por este motivo la solución de estilo Nash es posible; al igualar las destrezas delictivas de $j, \mathrm{y} k, \psi_{i}=\psi_{k}$ y sustituirlas en la ecuación (12), el equilibrio de Nash se transforma en:

$$
C^{\prime}\left(\psi_{i}\right)=\left(W_{1}-W_{2}\right) g(0) \quad i=\{j, k\}
$$

La decisión de un actor racional de entrar en un torneo con otros jugadores, es la diferencia entre ganar y perder el premio, la condición inicial es que la utilidad esperada sea positiva, tal como se expresa en la ecuación (13). Este comportamiento del mercado criminal permite la entrada de muchos actores, si la utilidad esperada es cero en su primer momento, forma la expectativa que al momento dos podrán llegar al premio, lo que determina que el mercado delictivo esté en expansión y sea dinámico. Expresado en otros términos una política criminal debe elevar los costos de oportunidad, con el propósito de desincentivar el acceso de los ciudadanos en el mercado delictivo. De esta forma se da un valor a la probabilidad $p$ de $50 \%$ y se remplaza en la ecuación (7), lo que implica que ganar el premio depende de los choques aleatorios, de esta forma la expresión se transforma en:

$$
(1 / 2)\left(W_{1}+W_{2}\right)-C\left(\psi_{i}\right) \geq 0
$$

Los ingresos del sector criminal provienen de la capacidad delictiva de los individuos y la forma en que se remuneren sus acciones, así la variable $\mathrm{V}$ es un proxy del precio de la accionar delincuencial; los premios se asumen como un costo para el sector, estos son incentivos que permiten desarrollar la capacidad delictiva, asumiendo que las capacidades de los individuos se igualen debido a una convergencia de factores, siendo así $\psi=\psi_{i}=\psi_{k}$. De esta manera se encuentra un equilibrio donde el beneficio es cero, esta condición explica porque el sistema criminal se renueva constante sin la necesidad de intervención de la figura de la planificación:

$$
\begin{gathered}
(1 / 2)\left(W_{1}+W_{2}\right)=V\left(\Psi_{i}+\Psi_{k}\right) \\
(1 / 2)\left(W_{1}+W_{2}\right)=V(\psi)
\end{gathered}
$$

$\mathrm{Al}$ sustituir la ecuación (16) en la expresión (7) se obtiene la mejor estrategia criminal, asumiendo que la probabilidad de ganar es del cincuenta por ciento:

$$
V(\psi)-C(\psi)
$$

El premio de un torneo maximiza la asignación de recursos en forma eficiente, al derivar la habilidad delictiva con respecto a los incentivos a ganar, resulta en:

$$
\left(V \psi-C^{\prime}\left(\psi_{i}\right)\right)\left((\partial \psi) /\left(\partial W_{i}\right)\right)=0
$$


$\mathrm{Al}$ igualar el anterior resultado, el costo marginal de desarrollar habilidades criminales es igual a la pérdida social de bienestar de la comunidad. Así la estructura de torneo permite la formación de bandas criminales especializadas, que emplean de forma eficiente los recursos que han desarrollado, por ello, la inercia delincuencial genera una industria criminal.

La forma de un torneo es similar al accionar criminal, al fijar un premio anticipado, los criminales incrementan su preparación delictiva lo que resulta en beneficios para el clúster criminal. La diferencia entre ganar o perder el premio, incrementa la productividad, elimina los individuos deficientes y atrae personas calificadas. Este comportamiento es similar a la teoría de la agencia, donde la firma desea incrementar sus beneficios y delega su función a otro agente, el problema resulta que el actor se apropia de los beneficios y desmejora a la firma, pero la estructura del torneo soluciona este problema porque la apropiación del premio por parte del ganador es inferior a la productividad que generó la firma durante el torneo, por esto el premio se acepta como un costo.

$\mathrm{Al}$ solucionar las ecuaciones (12) y (15), el problema de la agencia encuentra el equilibrio que maximiza la utilidad esperada con respecto a los incentivos.

$$
\begin{gathered}
W_{2}=-\left(\left(C^{\prime}\left(\psi_{i}\right)\right) /(g(0))\right)+W_{1} \\
\Rightarrow V \psi=\left(W_{1}-\left(\left(C^{\prime}\left(\Psi_{i}\right)\right) /(g(0))\right)+W_{1}\right)(1 / 2) \\
W_{1}=V \psi+\left(\left(C^{\prime}\left(\Psi_{i}\right)\right) /(2 g(0))\right)=V \psi+(V /(2 g(0))) \\
W_{1}=\left(\left(C^{\prime}\left(\psi_{i}\right)\right) /(g(0))\right)+W_{2} \\
\Rightarrow V \psi=\left(\left(\left(C^{\prime}\left(\psi_{i}\right)\right) /(g(0))\right)+W_{2}+W_{2}\right)(1 / 2)
\end{gathered}
$$

$$
W_{2}=V \psi-\left(\left(C^{\prime}\left(\psi_{i}\right)\right) /(2 g(0))\right)=V \psi-(V /(2 g(0)))
$$

Finalmente, la diferencia entre ganar y perder el torneo da como resultado un trade off, entre los ingresos del mercado delictivo, versus la inversión realizada en adquirir capacidades criminales, si la diferencia de habilidades entre un grupo de individuos se reduce $\mathrm{g}(0)$, y el incentivo se eleva sensiblemente; mientras ésta disminuye el premio se reduce. $\mathrm{Al}$ aumentar la capacidad delincuencial el premio es mayor, y la productividad criminal mejora.

$$
W_{1}-W_{2}=(V /(g(0)))
$$

\section{Evidencia empírica}

La tasa de homicidios de Bogotá se analiza como un índice donde el número de muertes violentas se divide por la cantidad de población de la ciudad de Bogotá y se multiplica por 100 mil, dando una medida que sirve para hacer comparaciones con otras ciudades, y que elimina el efecto inercial de la población.

La violencia bogotána se puede dividir en tres fases: la primera va de 1960-1978, durante este lapso de tiempo índice fue estable, a pesar de la migración rural que llegó a la ciudad producto de la política económica de Currie (Lauchlin, 1965, Vol. 24) y de la violencia partidista de la década del 50.

La segunda etapa de este indicador se encuentra a finales de la década de los ochenta y principios de los noventa. Durante esta fase, el crimen violento crece de forma exponencial, la explicación dada por violentólogos atribuyen este fenómeno al desarrollo del trafico de drogas ilícitas, que desembocó en la creación de cooperativas de exportación de alucinógenos. Esta explicación para la autora del presente escrito resulta simplista, porque parte de un lugar común, la serie de la figura 1 no permite inferir dicha explicación, no se sabe que parte de los crímenes 
son atribuibles a ese fenómeno y cuáles son fruto de otro tipo de fenómenos delictivos o sociales.

A partir de 1993 existe un punto de inflexión donde cambia la serie estructuralmente y la tendencia se invierte hasta el momento de escribir este artículo. Este descenso no ha sido explicado, las hipótesis parten de un cambio en la política pública de seguridad, la cual fortalece la estructura coercitiva del Estado, así como la implementación de programas comunitarios que obedecen a la Teoría de las ventanas rotas (Wilson \& Kelling, 1996).

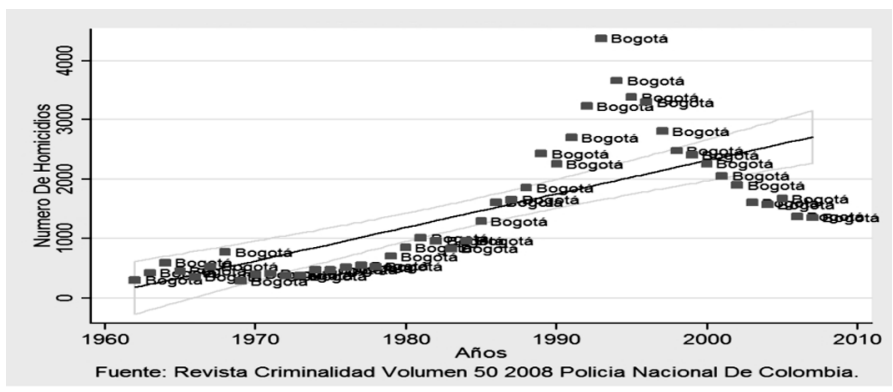

Figura 1. Serie histórica: homicidos Bogotá 1962-2008.

A partir de la encuesta de victimización realizada por el DANE en el $2003^{9}$, se presenta la descripción del comportamiento criminal. Ésta permite analizar el comportamiento del criminal de manera indirecta, gracias a que la victima proporciona información del modo de operar del delincuente. De manera que, la criminalidad oculta en Bogotá se ve expuesta por primera vez, igualmente permite cotejar la información oficial sobre seguridad y al final

9 La encuesta de victimización de 2003 tuvo un costo de 1600 millones de pesos, se realizó en coordinación con el Departamento Nacional de Planeación y la Secretaría de Gobierno de la Alcaldía Mayor de Bogotá, los resultados fueron vetados por el gobierno nacional y generó la destitución del director del DANE (Cesar Caballero). Al momento de solicitar el micro-datos de la encuesta, el DANE informó que la información no la tenían disponible; por lo tanto el análisis es descriptivo, (nota del autor). se puede evaluar la política pública de seguridad y reformular acciones que contrarresten el accionar criminal.

Los tres aspectos fundamentales de una encuesta de victimización es la denuncia ante las autoridades pertinentes sobre la comisión de delitos, esto revela el grado de eficacia del sistema de justicia e investigación criminalística, que logran disuadir de manera significativa las conductas delictivas.

El segundo punto de una encuesta de este tipo es analizar el comportamiento del delincuente, esto se logra con las preguntas del formulario, que se refieren a la forma en que se victimizó al ciudadano, descubriendo la lógica, motivaciones y forma de operar de un criminal, desafortunadamente, la no entrega de estas bases de datos a la comunidad académica, deja en una caja negra, este tipo de aspectos fundamentales al momento de formular políticas de seguridad acertadas. Finalmente, se indaga en la percepción de seguridad de los ciudadanos, esa información sólo resulta valiosa si se puede correlacionar con los dos ítems anteriores.

La primera parte de los datos indica que la población bogotána que ha sido víctima de un delito denuncia los hechos ante las autoridades en el caso de atraco callejero en un 29\%; para el caso de robo residencial se presentó denuncia en un 39,73\%; el robo de vehículos se denuncia casi en su totalidad, 96,37\%; el delito denominado paseo millonario, el cual se denomina secuestro simple, fue denunciado un 45,77\% y por último, los casos de extorsión fueron denunciados un $37 \%$.

Estas cifras indican que los datos oficiales sobre delitos en Bogotá están distorsionados, y que la confianza ciudadana es baja en relación con la respuesta de las autoridades para atacar los hechos delictivos de la ciudad. La segunda implicación de la política pública de seguridad, así como 
el desarrollo de estrategias y planes de acción contra la criminalidad, podrían estár mal direccionados debido a la ausencia de información. Cabe resaltar, cómo el robo de automotores se reporta casi en su totalidad, con este hecho se puede desarrollar un experimento natural de efectividad estatal para combatir el delito, lo que haría necesario tener la base de datos adecuada, como al momento de escribir el presente artículo no se posee dicha información, no es posible realizar dicha inferencia estadística.

Tabla 1. Tasa de no denununcias

\section{Delitos}

Tasa de no denuncias

\section{Hurto a personas}

Hurto a residencias

Hurto a vehículos

Riñas y golpes

Corrupción

$96,70 \%$

Paseo millonario

Extorsión $54,23 \%$ $62,88 \%$

Fuente: DANE, encuesta de victimización 2003.

A partir de esta información se desagrega la tasa de denuncias por estrato socio-económico, lo que arroja como resultado que a medida que aumenta el nivel de ingresos la tasa de denuncias crece; la correlación entre estrato y confianza en instituciones de seguridad y justicia es positiva, lo que indica como el nivel de ingresos incide en la gestión de las políticas de seguridad. Es de resaltar como el estrato dos presenta la mayor tasa de no denuncias; así mismo los estratos dos y tres exhiben las mayores tasas de victimización.

$\mathrm{Al}$ indagar por qué no se había denunciado el hecho delictivo, el resultado por estratos muestra que los niveles dos y tres presentan las mayores tasas de desconocimiento para colocar denuncias, desconfianza en las autoridades policiales, perdidas menores u otras razones, al mismo tiempo dichos estratos son los más victimizados y la tasa de denuncia es baja. Lo que forma una correlación entre los mecanismos de denuncia y los costos de ejecutar un hecho delictivo. La focalización de política pública en seguridad se debe concentrar en el estrato dos y tres, dirigida a mejorar los mecanismos de denuncia y el acercamiento de la comunidad con las autoridades de justicia y seguridad.

Tabla 2. Población victimizada por estrato

\begin{tabular}{|c|c|}
\hline Total & Victimización \\
\hline Estrato 1 & $8,51 \%$ \\
\hline Estrato 2 & $29,21 \%$ \\
\hline Estrato 3 & $45,96 \%$ \\
\hline Estrato 4 & $8,98 \%$ \\
\hline Estrato 5 & $4,72 \%$ \\
\hline Estrato 6 & $2,62 \%$ \\
\hline
\end{tabular}

Fuente: DANE, encuesta de victimización 2003.

Tabla 3. Tasa de no denuncia por estrato

\begin{tabular}{|c|c|}
\hline Total & No denuncia \\
\hline Estrato 1 & $70 \%$ \\
\hline Estrato 2 & $78 \%$ \\
\hline Estrato 3 & $68 \%$ \\
\hline Estrato 4 & $64 \%$ \\
\hline Estrato 5 & $45 \%$ \\
\hline Estrato 6 & $54 \%$ \\
\hline
\end{tabular}

Fuente: DANE, encuesta de victimización 2003.

Asimismo, las personas más victimizadas por género son los hombres que se encuentran entre los 18 y 25 años de edad y poseen grado de escolaridad secundaria, este grupo social presenta los menores índices de no denuncias. 
Tabla 4. Victimización por género

\section{Mujer}

\section{Hombre}

$40.35 \%$

$59.65 \%$

Fuente: DANE, encuesta de victimización 2003.

Esta caracterización permite inferir que un delincuente es un individuo profesional que ataca victimas vulnerables, que no presentan denuncia ante las autoridades competentes a fin de judicializar los actos de los que han sido víctimas, lo que permite reducir los costos en que incurre el delincuente, aumentar los beneficios y reducir los riesgos de su actividad criminal.

El modelo teórico de comportamiento criminal, desarrollado anteriormente, explica el accionar delictivo en Bogotá, permite inferir como la productividad criminal asciende; en primer término se ataca de forma masiva poblaciones vulnerables con recursos medios, que no inician acciones defensivas o agresivas contra los ofensores. Se ataca en menor medida a estratos altos, pero cuando se ejecutan acciones criminales, son de alto valor y agravian de forma sensible a la sociedad, pues se caracteriza por el grado de tecnicismo delictivo, que comprueba la teoría del torneo criminal.

\section{Conclusión}

La criminalidad como acción racional existe y crece en la ciudad, debido en mayor medida al desconocimiento del fenómeno, en consecuencia se incurre en errores al momento de tomar decisiones de política pública de seguridad. Las políticas establecidas para combatir la criminalidad no tienen una base de conocimiento científico; es necesario destinar urgentemente, recursos que permitan estudios e investigaciones formales que diagnostiquen el estado de la actividad delictiva en Bogotá. Los cambios metodológicos que se han presentado en las estadísticas nacionales requieren de un estudio técnico que estime las series, con el objeto de minimizar las discrepancias estadísticas, y que afectan la calidad de los resultados obtenidos en la eficacia de las políticas públicas de seguridad.

Un marco científico permite una visión clara de los hechos criminales en la ciudad, y se traduce en un diseño de política que permita reducir drásticamente la criminalidad, así como su correcto seguimiento y evaluación. Como se demostró en este trabajo, la criminalidad es una actividad racional que actúa de acuerdo a la probabilidad de ser aprehendida y castigada, asume la forma de un torneo que posibilita eliminar los elementos menos aptos para el crimen y desarrolla actividades que mejoran la productividad criminal. Esto evidencia un aprendizaje criminal donde las políticas coercitivas deben poseer un componente estratégico importante, el atacar el punto álgido de la cadena criminal eleva los incentivos para que la base se siga ampliando, de esta forma se malgastan recursos valiosos de seguridad.

La evaluación de la criminalidad es un asunto pendiente en la agenda política de defensa y seguridad, la escasa información estadística así como las trabas para acceder a ella no permite formular planes de acciones eficientes, este documento desarrolló una herramienta que requiere una validación empírica, para ello es necesario abundante material estadístico.

Finalmente, el descenso criminal de Bogotá a partir de 1993 no es un hecho aislado en el país, y se desmiente el mito que la cultura ciudadana es el componente que permitió dicho declive, lo que se traduce en que la teoría de las ventanas rotas es un explicación teórica que no resiste una prueba empírica rigurosa, así el comportamiento criminal de la ciudad no ha recibido una explicación acertada por parte de los investigadores criminológicos, 
lo que plantea este escrito es desarrollar dicho análisis con herramientas adecuadas para tal fin.

\section{Referencias}

Acero, H. et ál. (1997). Políticas saludables para la seguridady la convivencia. Bogotá: Alcaldía Mayor de Bogotá.

Aguirre, K. \& Restrepo,J. (2005). Aproximación a la situación de violencia e inseguridad en Bogotá D.C. Bogotá: CERAC.

Martin, G. \& Ceballos, M. (2004). Bogotá: anatomía de una transformación, politicas de seguridad ciudadana. Bogotá: Pontificia Universidad Javeriana. USAID, Georgetown.

Becker, G. (1968). Crime and Punishment: an Economic Approach. In The Fournal of Political Economy. Chicago.

Becker, G. (1992). Nobel Lecture: the Economic Way of Looking at Life. Chicago: Department of Economics, University of Chicago.

Bentham, J. (1970). The Principles of Morals and Legislation. London: edited by J.H. Burns and H.L.A Hart, 1st edition. The Athlone Press (1970). The Athlone Press; Oxford: Clarendon Press.

Casas, P. \& González, P. (2005). Políticas de seguridad y reducción del homicidio en Bogotá: mito y realidad. En Seguridad urbana y policía en Colombia. Bogotá: Fundación Seguridad y Democracia.

Cámara de Comercio de Bogotá (2008). La encuesta de percepción y victimización Bogotá y las localidades. Bogotá: Dirección de Seguridad y Convivencia Vicepresidencia de Gestión Cívica y Social.

DANE (2003). Encuesta de victimización. Bogotá.
De León, I. et ál. (2003). Homicidio e intención letal: un estudio exploratorio de heridas mortales a partir de los protocolos de necropsia en Bogotá. Bogotá: Método Grupo Transdisciplinario de Investigación en Ciencias Sociales, Borradores de Método, área de crimen y conflicto.

De León, I. \& Forero, L. (2004). Una descripción de las armas de fuego homicidas en Bogotá para el año 2002 y una propuesta para aumentar el costo del servicio de homicidio. Bogotá: Método Grupo Transdisciplinario de Investigación en Ciencias Sociales, Borradores de Método, área de Crimen y Conflicto.

Di Tella, R. \& Schargrodsky, E. (2004). Do Police Reduce Crime: Estimates Using the Allocation of Police Forces After a Terrorist Attack. In American Economic Review (94).

Donohue, J. et ál. (2000). The Impact of Legalized Abortion on Crime. NBER WP 8004.

Gaviria, A. et ál. (2008). The Cost of Avoiding Crime: the Case of Bogotá. En Borradores de Economía, No. 508. Bogotá: Banco de la República.

Jaén, P. \& Dyner, R. (2002). Políticas sostenibles para la prevención y la disminución de la criminalidad en Colombia. Bogotá: Instituto de Sistemas y Ciencias de la Decisión, Universidad Nacional de Colombia.

Lazear, E. \& Rosen, S. (1981). Rank-Order Tournaments as Optimum Labor Contracts. In Fournal of Political Economy, Vol. 89, No. 5.

Lauchlin, C. (1965). Ideas básicas sobre la aceleración del desarrollo: ¿tiene solución la crisis económica? Conferencia con motivo del premio obtenido por Accelerating Development. En Economía Colombiana, Vol. 24. 
Levitt, D. \& Dubner, J. (2006). Freakonomics. Ediciones B.

Levitt, D. \& Sudhir, A. (2000). An Economic Analysis of A Drug-selling Gang's Finances. In Quarterly fournal of Economics, Vol. 115.

Llorente, M. et ál. (2000). Violencia homicida en Bogotá: más que intolerancia. Bogotá: Centro de Estudios sobre Desarrollo Económico, Facultad de Economía; Paz Pública: Programa de Estudios sobre Paz, Justicia y Violencia, Universidad de los Andes.

Llorente, M. et ál. (2001). Violencia homicida y estructuras criminales en Bogotá. Bogotá: Centro de Estudios sobre Desarrollo Económico, Facultad de Economía; Paz Pública: Programa de Estudios sobre Paz, Justicia y Violencia, Universidad de los Andes.

Moreno, J. (2005). Impacto de Transmilenio en el crimen de la Avenida Caracas y sus vecindades. Bogotá: Documento Cede, Universidad de los Andes.

Nash, J. (1950). Equilibrium points in person games. In The National Academy of Sciences, USA 36.

Parada, O. (2007). Por el cual se reviste al Alcalde Mayor de Bogotá, de facultades extraordinarias para aumentar el pie de fuerza policial en el Distrito Capital y se dictan otras disposiciones. Bogotá: Proyecto de Acuerdo No. 425, Concejo de Bogotá D.C.

Peñalosa E. (2000). La rehabilitación de la ciudad de Bogotá 1998-2000. Propuesta de un modelo de ciudad. Bogotá: Alcaldía Mayor de Bogotá.
Restrepo, M. \& Moreno, A. (2007). Bogotá: ¿más crimen?, ¿más miedo?”, desarrollo y sociedad. Bogotá: Universidad de los Andes.

Rivas, A. (2003). Una década de políticas de seguridad ciudadana en Colombia. Bogotá: Fundación Seguridad \& Democracia.

Rubio, M. (1998). Criminalidad urbana en Colombia. Bogotá: Centro de Estudios sobre Desarrollo Económico, Facultad de Economía; Paz Pública: Programa de Estudios sobre Paz, Justicia y Violencia, Universidad de los Andes.

Sánchez, F. et ál. (2003). ¿Garrote o zanahoria? Factores asociados a la disminución de la violencia homicida y el crimen en Bogotá, 1993-2002. Bogotá: Documento Cede, Universidad de los Andes.

Sánchez, F. \& Núñez, J. (2001). Determinantes del crimen violento en un país altamente violento: el caso de Colombia. Bogotá: Documento Cede, Universidad de los Andes.

Smith, A. (1994). Investigación sobre la naturaleza y causas de la riqueza de las naciones. México: Fondo de Cultura Económica.

Wilson, J. (1996). The Police and Neighborhood Safety: Broken Windows. John F. Kennedy School of Government Harvard. 